\title{
Einführung in die Thematik von (großen) Rutschungen mit dem Schwerpunkt der Zustands- und Sicherheitsbeurteilung
}

\author{
Roman Marte \\ Institut f. Bodenmechanik und Grundbau, Technische Universität Graz, Graz, Österreich \\ Eingegangen 20. Oktober 2016; angenommen 26. Oktober 2016; online publiziert 16. November 2016
}

Zusammenfassung: Im gegenständlichen Beitrag wird in Kurzform in die Thematik von Rutschungen im Lockergestein eingeführt. Dabei wird das Augenmerk auf die Zustands- und Sicherheitsbeurteilung (großer) Rutschungen gelegt. Im Detail wird weiters auf rechnerische Ansätze als Grundlage der Sicherheitsbewertung wie auch möglicher Sicherungsansätze eingegangen. In Kurzform wird sodann noch die Bedeutung messtechnischer Erkundungs- und Überwachungsmaßnahmen diskutiert und einige allgemeine Gedanken zur Planung von Sicherungsmaßnahmen ausgeführt.

Schlüsselwörter: Rutschung, Zustandsbeurteilung, Standsicherheitsuntersuchung, Risikobewertung, Beobachtungsmethode, Messtechnik

Introduction into the Topic of (Big) Landslides with Focus to the As-is State Evaluation and Risk Analysis

Abstract: In this paper a short introduction into the topic of landslides in soils is presented. Main focus is given to the evaluation of the as-is state and the risk analysis of (big) landslides. In detail analytical assumptions as basis for the safety assessment as well as remediation aspects are discussed. Further on the importance of the observational method for landslide problems is highlighted and some general remarks on the design of remediation measures are presented.

Keywords: Landslide, As-is state evaluation, Stability analysis, Risk analysis, Observational method,

Measurement technique

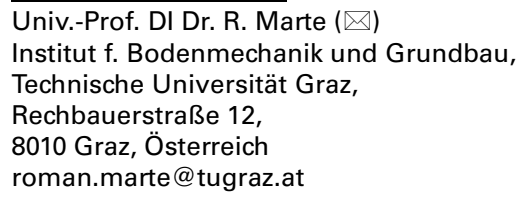

\section{Einführung}

Massenbewegungen als Überbegriff aller Formen von gravitativ bewegten Fest- oder Lockergesteinsmassen sind eine der häufigsten und gleichzeitig vielfältigsten Problemstellungen in der Geotechnik. Eine typische Untergliederung von Massenbewegungen erfolgt nach der Bewegungsform, wobei z.B. in [1] eine Unterscheidung in Gleiten, Kippen, Knicken und Abscheren, Fallen, Fließen sowie Driften und Kriechen vorgenommen wird. Bedient sich die Schwerkraft der Mithilfe von Transportmitteln wie Wasser (z.B. Mure), Eis (z.B. Blockgletscher) oder Luft ("Bodenverflüssigung mit Luft “), wird auch der Begriff Massentransport oder Erosion verwendet.

Rutschungen sind eine Sonderform von Massenbewegungen, bei der kein „Fallen“ (Bergsturz, Felssturz) stattfindet. Die größten bekannten Rutschungen sind submariner Natur. Die Storegga-Rutschung vor der Westküste Norwegens weist beispielsweise ein Volumen von mehr als $5000 \mathrm{~km}^{3}$ mit einer Länge von ca. $800 \mathrm{~km}$ auf [2]. Rutschungen sind in Abhängigkeit ihrer Lokalität, Größe und spezifischen Erscheinungsform wegen ihres Schadenspotentials von besonderer volkswirtschaftlicher Bedeutung. Insbesondere auch im Zusammenhang mit möglichen Klimaveränderungen (Änderung der Temperatur- und Niederschlagscharakteristik ganzer Regionen) wird ihnen zukünftig eine noch größere Bedeutung zukommen, da auch bis dato vermeintlich „stabile“ Hänge Veränderungen erfahren können. Einleitend angemerkt wird auch, dass es im Bereich der Geotechnik wenige Problemstellungen gibt, die eine vergleichbare Interdisziplinarität aufweisen und erfordern, wie dies für Rutschungen der Fall ist. Neben den für Geotechniker/Innen vordergründigen Fachbereichen wie Geologie, Bauwesen und Messtechnik spielen hierbei auch Fachbereiche wie z.B. Meteorologie, Geographie, Infrastrukturbau und -erhaltung, besonders aber auch behördliche und oftmals auch politische Gesichtspunkte eine entscheidende Rolle. 
Im gegenständlichen Beitrag wird der Versuch unternommen, in Kurzform eine Einführung in die Thematik von Lockergesteins-Rutschungen und den Umgang mit deren vielfältigen Erscheinungsformen im alpinen Raum zu geben. Die Einschränkung auf den alpinen Raum dient in erster Linie zur Themabegrenzung, da auf eine Vielzahl typischer Rutschungen wie beispielsweise Rutschungen im Zusammenhang mit Vulkanen, vulkanischen Böden, submarine Rutschungen etc. nicht Bezug genommen wird. Die in diesem Beitrag kurz angeschnittenen Themenbereiche wie einige kurze Vorbemerkungen zu Rutschungen, Risiko- und Sicherheitsbewertung, Modellbildung und rechnerische Ansätze, messtechnische Erkundungsund Überwachungsmaßnahmen sowie einige allgemeine Anmerkungen zu den Grundlagen für Sicherungs- und Sanierungsmaßnahmen stellen gleichzeitig auch die Grobgliederung des Beitrages dar. Angemerkt wird weiters, dass es sich im gegenständlichen Beitrag um eine einfach gehaltene Einführung in die Thematik von Rutschungen handelt, die aufgrund des vorgegebenen Umfangs keine tiefergehenden Betrachtungen einzelner Detailthemen erlaubt.

\section{Kurze Vorbemerkungen zum Thema von Rutschungen}

Bei der Diskussion von Rutschungen, d.h. der genaueren Erfassung von deren Einflussgrößen, der Charakterisierung einer Rutschung, der Risiko- bzw. Sicherheitsbeurteilung sowie der eventuellen Untersuchung möglicher Sicherungsansätze wird i.A. versucht, zwischen „Ursache“ (source) und "Auslöser" (trigger) zu unterscheiden. Als Ursachen werden i.A. die zumeist längerfristig vorhandenen Randbedingungen verstanden, die zu einer grundsätzlichen Rutschungsgefährdung an einem gegebenen Standort führen. Als Beispiel für eine mögliche Ursache sind die geologischen Verhältnisse an einem Standort zu nennen. Als Auslöser werden sodann natürliche oder anthropogene Einflüsse, wie z.B. Starkniederschläge (= natürlich), Geländeabtrag im Zuge von Baumaßnahmen und damit verbundene Versteilung (= anthropogen) verstanden, die letztlich zur Auslösung eines Rutschungsereignisses oder eines Bewegungsschubes führen (können).

Die Unterscheidung nach Ursachen und Auslösern ist hilfreich, wenn es darum geht, die wesentlichen Einflussfaktoren einer Rutschung zu erkennen und deren Einfluss zu quantifizieren. Die Analyse und Gewichtung der für eine Rutschung maßgebenden Ursachen und Auslöser ist die wesentliche Voraussetzung für eine brauchbare Risikobewertung, wie auch Grundlage jeglicher Form einer technisch und wirtschaftlich optimierten Sicherungsmaßnahme. Bei nicht ausreichender Kenntnis dieser Grundlagen besteht die Gefahr einer "falschen Risikobewertung" wie auch einer, auf die Zieldefinition bezogenen, unter- bzw. überdimensionierten Sicherungsmaßnahme, die über kurz oder lang in beiden Fällen zu (wesentlichen) Mehrkosten führen wird.

Kurz angemerkt wird noch, dass der Begriff des Geotechnikers einerseits weibliche und männliche Fachpersonen umfassen soll und im Weiteren darunter weniger die auf eine Person vereinte Kompetenz der verschiedenen Fachbereiche der Geologie und des Bauingenieurwesens (Bodenmechanik, Felsmechanik, Grundbau), sondern vielmehr die Zusammenarbeit und Ergänzung dieser Fachbereiche in einem Team, die erfahrungsgemäß zu den qualitativ besten Ergebnissen führt, verstanden werden soll.

\section{Risiko- und Sicherheitsbewertung von Rutschungen}

Bevor auf ausgewählte „technische“ Details zu dieser Fragestellung eingegangen wird, werden zwei wesentliche Fragen beleuchtet, die i.A. im Zuge der Risikobewertung von Rutschungen geklärt werden müssen:

1. Was kann passieren? = Risikoanalyse (Risk Analysis)

2. Was darf passieren? = Risikobewertung (Risk Assessment)

Die erste Frage der Risikoanalyse, die auch die Effektivität bzw. Sicherheitserhöhung durch mögliche Sicherungsmaßnahmen für eine Rutschung umfasst, liegt gänzlich im Aufgaben- und Verantwortungsbereich der Geotechniker. Die zweite Frage nach der Risikobewertung - „Was darf passieren"- bedarf grundsätzlich anderer Personen und Experten wie z.B. Eigentümer, Politiker oder Behörden, sonstige Fachexperten (z.B. Konstruktive oder sonstige Ingenieure, um die Vulnerabilität von Bauwerken oder z.B. Infrastrukturanlagen zu bewerten). D.h. die Antwort auf die Frage "Was darf passieren" liegt nicht im Verantwortungsbereich der Geotechniker, sondern des oben genannten Personenkreises [3]. Diese haben die Entscheidung zu treffen, ob die zuvor von Geotechnikern abgeschätzten Risiken „akzeptierbar" sind oder nicht. In der Praxis wird diese Unterscheidung oftmals nicht (rechtzeitig) klargestellt, was zu undefinierten und in Folge oftmals missverstandenen Entscheidungs- und Verantwortungskompetenzen führt bzw. führen kann. Eine entsprechende Klarstellung ist deshalb ein wichtiger erster Schritt bei der gemeinsamen Bearbeitung eines größeren Rutschungsprojekts.

Die Beurteilung des Sicherheitszustandes einer Rutschung bzw. die Beurteilung einer Veränderung hin zu einem kritischen (Versagens-)Zustand kann auf verschiedenen Ebenen erfolgen. Einerseits kann dies in klassischer Form durch rechnerische Ansätze mit dem Ergebnis einer Sicherheitszahl stattfinden. Hierzu wird auf Abschn. 5 verwiesen. Andererseits kann eine derartige Beurteilung über messtechnisch erfasste Veränderungen bzw. Entwicklungen charakteristischer Größen wie absolute Verschiebungsbeträge, Verschiebungsraten, kritische Porenwasserdrücke etc. erfolgen. Sehr häufig werden als Kriterium Bewegungsraten und deren zeitliche Entwicklung herangezogen. In kritischen Fällen, bei denen sich ein Versagen durch eine Zunahme der Bewegungsrate (Bewegungsgeschwindigkeit) der Rutschung andeutet und ein duktiles Verhalten vorliegt, ist beispielsweise die Darstellung der inversen Bewegungsrate 1/v über die Zeit hilfreich. In Abb. 1 ist eine derartige Darstellung nach [3] beispielhaft angeführt. Die 


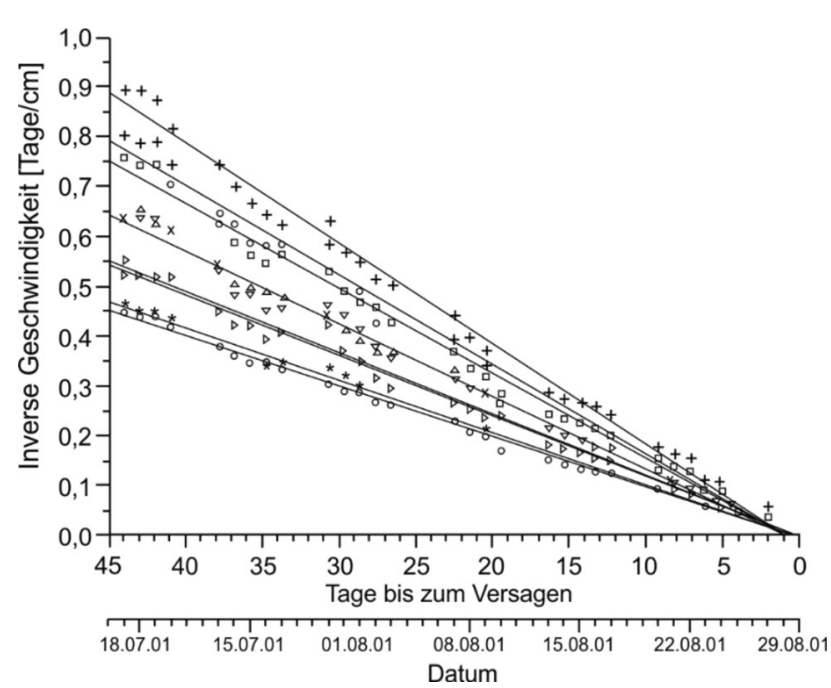

Abb. 1: Darstellung der inversen Geschwindigkeit einer Rutschung über die Zeit zur Abschätzung des zu erwartenden Versagenszeitpunktes. Schematisiert nach [3]

Verlängerung der invers aufgetragenen Geschwindigkeitsentwicklung und der Schnittpunkt dieser "Geraden“ mit der Nullachse $(v=\infty$ bzw. $1 / v=0)$ zeigt den "theoretischen" Versagenszeitpunkt an.

Eine weitere Möglichkeit der Zustandsbewertung kann über die Entwicklung von Schäden an wichtigen Bauwerken oder Infrastrukturanlageteilen auf oder im Einflussbereich einer Rutschung erfolgen. Ein solcher Ansatz wird zumeist dann gewählt, wenn eine oder mehrere Anlageteile den Hauptfokus bzw. das Hauptgefährdungspotential darstellen. Beispielhaft sei eine Eisenbahnstrecke, die eine Rutschung quert, genannt. Die Überwachung (z.B. Verschiebungsmessung der Gleise) gibt Auskunft über die Nutzbarkeit bzw. allenfalls erforderliche Einschränkungen - wobei als "Versagen" ein als unzulässig definierter Verformungszustand der Gleise definiert werden kann/muss.

Bei der Beurteilung der "Sicherheit" bzw. des vorherrschenden "Risikos", das von einem Rutschungskörper ausgeht, also der Frage nach dem „Was kann passieren?”, sind aus technischer Sicht im Weiteren folgende Aspekte von Bedeutung (Auflistung beispielhaft und nicht vollständig):

Kubatur und Tiefe einer Rutschung (bzw. "mobilisierbare Kubatur"): Sowohl die Kubatur wie auch die Tiefe einer Rutschung haben i.A. Einfluss auf das Bewegungsverhalten einer Rutschung und geben die Möglichkeiten bzw. Grenzen „sicherheitserhöhender", technischer Maßnahmen für einen Rutschungskörper unter Berücksichtigung technischwirtschaftlicher Randbedingungen vor. Die Kubatur einer Rutschung ist u.A. von Bedeutung, da die stützenden Kräfte zur Erhöhung der "Standsicherheit" des Rutschungskörpers in etwa proportional zur Rutschungskubatur sind. Dies trifft dann zu, wenn treibende Kräfte, wie z.B. Porenwasserdrücke in Gleitflächen, Strömungskräfte im Gleitkörper oder das Volumen des Rutschungskörpers selbst im Zuge der Sicherungsarbeiten nicht durch entsprechende Maßnahmen (wie z.B. wirksame Porenwasserdruckentspannungs- bzw. Entwässerungsmaßnahmen oder den Abtrag eines Teils des Rutschungskörpers) reduziert werden kön- nen. Ansonsten kann davon ausgegangen werden, dass für die "gewünschte" Erhöhung der Standsicherheit eines Rutschungskörpers um beispielsweise $\Delta \eta=5 \%$-Pkte für die doppelte Rutschmasse in etwa der doppelte Widerstand (also z.B. die doppelte Anzahl von Ankerkräften) aufzubringen ist. Als sehr grober Erfahrungswert kann für einen (im alpinen Raum typischen) Rutschungskörper mit ca. 0,5 Mio $\mathrm{m}^{3}$ bewegter Masse für die Erhöhung der rechnerischen Sicherheitszahl $\Delta \eta=5 \%$-Pkte von ca. 200 Freispielanker á 1000 kN Vorspannkraft ausgegangen werden. U.A. beeinflusst die Neigung der Anker die tatsächliche sicherheitserhöhende Wirkung, weshalb es sich wie angeführt um einen sehr groben Erfahrungswert handelt [4,5]. Was sich aus einem derartigen Erfahrungswert ableiten lässt, ist die Grobabschätzung, bis zu welcher Kubatur eine relevante Erhöhung der Sicherheitszahl für eine Rutschung aus technisch-wirtschaftlichen Gesichtspunkten möglich bzw. leistbar ist.

Die "mobilisierbare Kubatur" einer Rutschung ist für Rutschungstypen von besonderer Bedeutung, die nach der Auslösung der Rutschung - i.A. in Zusammenhang mit Wasser - zu schuttstromartigem Verhalten (Schuttströme, Muren) führen (können), wobei eine deutlich größere „Kubatur" mobilisiert werden kann, als es dem ursprünglichen Rutschkörper (Abrisskörper) entspricht. Derartige Rutschungstypen bzw. Rutschungskörper sind hinsichtlich ihres Entwicklungspotentials und Gefährdungspotentials wesentlich schwieriger einzuschätzen als klassische Rutschungskörper, deren Volumen i.A. annähernd gleich bleiben.

Reichweite einer Rutschung: Im Zusammenhang mit dem zuletzt genannten schuttstromartigen Rutschungstyp ist festzustellen, dass jene Rutschungen, die sich am weitesten von ihrem eigentlichen Ursprungsort bzw. Auslösungsbereich wegbewegen, i.A. auch jene mit dem größten Risiko- bzw. Schadenspotential sind [3]. Die Prognostizierbarkeit eines derartigen Verhaltens, dass aus "klassischen Rutschungskörpern" Schuttströme hervorgehen bzw. hervorgehen können, ist zumeist schwierig. Da die Entwicklung einer "klassischen Rutschung“ zu einem schuttstromartigen Verhalten hin zumeist einen entsprechend großen Sättigungsgrad im Untergrund voraussetzt oder aber in Verbindung mit Extremniederschlägen auftritt, ist die Entstehung (zumindest im alpinen Raum) eher mit seichten Rutschungstypen verbunden, die im Zuge ihrer Entwicklung jedoch deutlich größere Massen (als die initiale Rutschung selbst) mobilisieren können.

Ein weiterer wesentlicher Gesichtspunkt bei der Risikobewertung von Rutschungen ist ihre (mögliche) Geschwindigkeit. Typische Geschwindigkeiten von "Rutschungen“ reichen von "kriechförmig (= extrem langsam)“ ablaufenden Bewegungen ( $\mathrm{mm} / \mathrm{Jahr}$ bis $\mathrm{cm} / \mathrm{Jahr}$ ), über sehr langsam bis langsam ( $\mathrm{m} / \mathrm{Jahr}$ bis $\mathrm{m} /$ Monat) und schnell bis hin zu sehr schnell ablaufenden Bewegungen, wie dies z.B. bei Muren mit mehreren $\mathrm{m} / \mathrm{sec}$ der Fall ist [1]. Die Möglichkeit, auf Rutschungen entsprechend zu "reagieren“ und Schutz- sowie Evakuierungmaßnahmen einzuleiten, ist dabei umgekehrt proportional zur Geschwindigkeit einer „Rutschung“. Das Schadenspotential unter Berücksichtigung der Kubatur der bewegten Masse nimmt i.A. ent- 
sprechend mit der Geschwindigkeit der Massenbewegung zu.

Für die Risikobewertung wie auch den Umgang mit Rutschungen weiters von Bedeutung ist die Unterscheidung, ob ein mehr oder weniger unverbauter Rutschungskörper vorliegt, der z.B. unterhalb gelegene Infrastrukturanlagen oder Siedlungsräume bedroht, oder ob es sich um einen besiedelten bzw. bebauten Rutschungskörper handelt. Im alpinen Raum gibt es eine Mehrzahl von Großmassenbewegungen, bei denen ein Teil der bewegten Masse besiedelt ist. Beispielsweise liegt der östliche Bereich von Bad Gastein auf dem Talzuschub des Graukogels [6, 7], oder großflächige Bereiche des Nassfelds in Kärnten sind Teil der Schlanitzenalmgleitung [8]. Typische Bewegungsraten derartiger besiedelter Großmassenbewegungen liegen im $\mathrm{mm}$ - bis mehrere $\mathrm{cm}$-Bereich pro Jahr. Da es sich hierbei in vielen Fällen um historisch gewachsene Siedlungsräume handelt, stellt sich die Frage der generellen Bebaubarkeit zumeist nicht (mehr). Sehr wohl jedoch sind für geplante, insbesondere größere Neubauten (z.B. Hotelanlagen) besondere Untersuchungs- und Überwachungsmaßnahmen sowie zumeist auch besondere bauliche Maßnahmen erforderlich, um eine möglichst lange Lebens- und Nutzungsdauer sicherzustellen. Dabei sind weniger die Gesamtbewegungen des Hanges als vielmehr die zu erwartenden differentiellen Bewegungen am Bauplatz für die Beurteilung der "Bebaubarkeit", allenfalls erforderlichen Sondermaßnahmen wie auch der zu erwartenden Lebensdauer und Nutzbarkeit maßgebend. Die Problemstellung der Errichtung von Neubauten und deren weitere Erhaltung auf großen Massenbewegungen (Talzuschüben) ist in den letzten Jahren auch durch den touristischen Ausbau von Gebirgsregionen - beispielsweise durch die Neuerrichtung einer großen Zahl von Beschneiungsteichen - aktueller geworden [9].

\section{Modellbildung und rechnerische Ansätze}

Für Rutschungen gibt es je nach Bewegungsform und insbesondere Bewegungsgeschwindigkeit unterschiedliche rechnerische Ansätze. Während schnell bis sehr schnell ablaufende Bewegungsformen i.A. Stoffgesetze für flüssigkeitsähnliches Verhalten erfordern, werden für langsam bis extrem langsam ablaufende Bewegungsformen i.A. „klassische Berechnungsansätze" wie z.B. Lamellenverfahren mit Mohr-Coulomb Versagenskriterium oder hochwertigere Stoffgesetze mit viskosen Ansätzen verwendet [10, 11]. Auf die letztgenannten Typen von Rutschungen, also langsam bis extrem langsam ablaufende Rutschungen, wird im Folgenden etwas genauer eingegangen.

Das Ergebnis oben genannter Standsicherheitsanalysen (für langsam ablaufende Rutschungstypen) ist der sogenannte Sicherheitsfaktor (Factor of Safety), zumeist mit $\eta$ bezeichnet. Dieser ist der Ausdruck des Verhältnisses von stabilisierenden Kräften z.B. zufolge vorhandener Scherfestigkeit zu destabilisierenden Kräften wie z.B. Gravitationsoder Erdbebenkräften. Was diese Sicherheitszahl jedoch nicht (bzw. nur indirekt) ausdrückt, ist die (quantifizierte) „Wahrscheinlichkeit für das Versagen“ einer Rutschung [3].
Die Frage nach der Versagenswahrscheinlichkeit ist aber zumeist eine der wesentlichen Fragen, welche seitens einer Behörde an Geotechniker gestellt wird: "Mit welcher Wahrscheinlichkeit ist damit zu rechnen, dass es zu einem Versagen des Hanges und in Folge zu diesen und jenen Schäden innerhalb eines Jahres oder fünf Jahren kommen wird?" Obwohl es in gewissen Programmpaketen die Möglichkeit von probabilistischen Berechnungsansätzen gibt, bleibt die Prognosemöglichkeit über die Versagenswahrscheinlichkeit einer Rutschung zumindest derzeit noch eine sehr schwierige Aufgabe. Sofern seitens des Auftraggebers quantitative Aussagen gewünscht werden, werden solche in der Ingenieurpraxis deshalb zumeist nicht auf Berechnungsergebnissen oder probabilistischen Untersuchungen alleine aufbauend angegeben. Bei Anwendung des Sicherheitsfaktors zur Beurteilung des Sicherheitszustandes einer Rutschung wird davon ausgegangen, dass bei einem Wert von $\eta=1,0$ das Grenzgleichgewicht erreicht ist, d.h. dass für Sicherheitszahlen $\eta<1,0$ ein Versagen eintritt. Offen bleibt, wie sich dieses Versagen genau darstellt bzw. wie es definiert wird. Generell ist nicht einheitlich festgelegt, ob bei Unterschreiten dieses Grenzwertes ein Rutschhang dann zur Gänze abgleitet oder ob z.B. eine bestimmte kritische Bewegungsrate erreicht wird

Eine Besonderheit von Rutschungen innerhalb des Fachbereichs des Bauingenieurwesens ist, dass die normenseitig geforderten (Stand-)Sicherheitsniveaus nur bedingt und oftmals gar nicht eingehalten werden können. Dies trifft insbesondere für große Rutschungskubaturen mit mehreren $100.000 \mathrm{~m}^{3}$ oder mehreren Millionen $\mathrm{m}^{3} \mathrm{zu}$. Bei diesen können oftmals aus technischen und/oder wirtschaftlichen Gründen keine ausreichend umfangreichen Sicherungsmaßnahmen hergestellt werden, um normgemäße Sicherheitsniveaus zu erreichen [12]. Ziel von technischen Maßnahmen ist in solchen Fällen eine geringfügige Erhöhung der "rechnerischen Sicherheitszahl ๆ, um einerseits ein plötzliches (unangekündigtes) Versagen mit "ausreichender Sicherheit" ausschließen zu können und die mittleren, jährlichen Bewegungsraten der Rutschung i.A. um einen Faktor 10 bis 100 verringern zu können, um Schadensentwicklungen an Bauwerken oder Infrastrukturanlagen zu verzögern.

Bei der Beurteilung der oben erwähnten rechnerischen Sicherheitszahl einer Rutschung im Lockergestein wird zumeist auf das Mohr-Coulomb'sche Bruchkriterium zurückgegriffen, wobei die maßgebenden Scherparameter durch den Reibungswinkel $\phi^{\prime}$ und die Kohäsion c' gegeben sind. Während diese Scherparameter in vielen Fragestellungen der Geotechnik durch geeignete Erkundungsmethoden und Laborversuche ermittelt und den weiteren Berechnungen zugrunde gelegt werden, ist dies bei vielen (insbesondere größeren) Rutschungen oftmals schwierig und nicht zielführend. Die Schwierigkeit besteht z.B. darin, dass die Rutschungsflächen bzw. -zonen in vielen Fällen (vorab) nicht bekannt sind und speziell bei großen Rutschungen sehr heterogene Verhältnisse entlang dieser Rutschungsflächen bzw. -zonen vorherrschen können, womit mittels Einzelversuchen kaum charakteristische Werte, welche für die gesamte Rutschungsfläche zutreffend sind, festgestellt werden können. Weiters ist es oftmals auch nicht bekannt, 


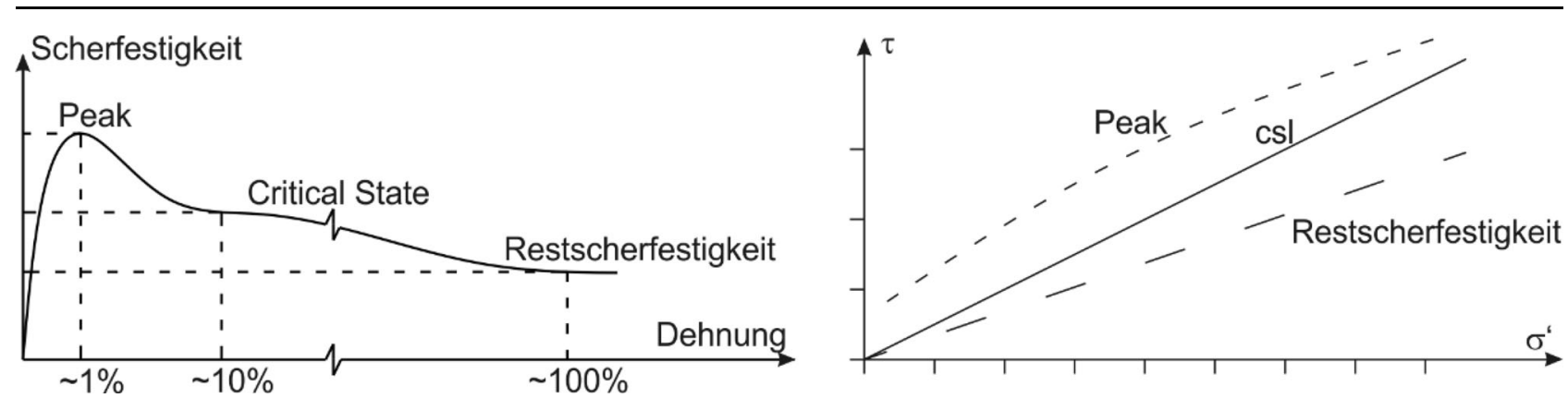

Abb. 2: Schematische Darstellung des Peak Werts, Critical State Wertes und der Restscherfestigkeit von Böden nach Atkinson 2013 [13] (Abb. 2a) bzw. Wood 2007 [15] (Abb. 2b)

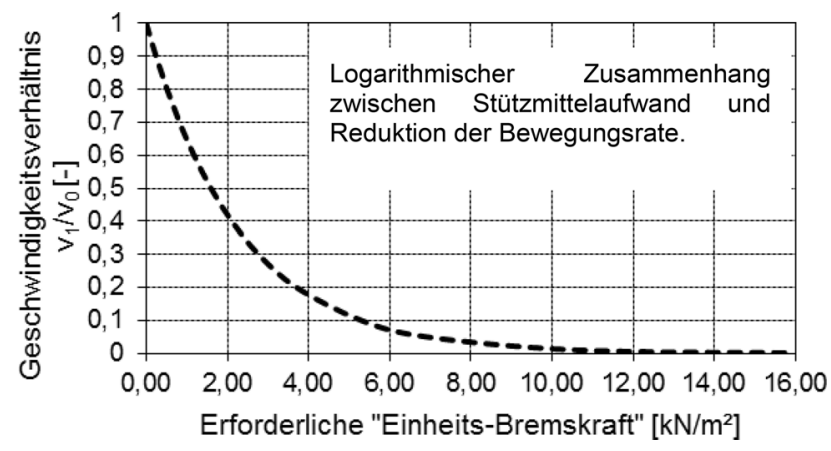

Abb. 3: Zusammenhang zwischen reduzierter Bewegungsrate und der erforderlichen Einheitsbremskraft/m² Rutschfläche nach Lippomann [10]

inwieweit bereits stattgefundene Bewegungen zu einem Abfall der Scherfestigkeit auf den "Residualwert" (=Restscherfestigkeit) in der Gleitzone geführt haben [12-14]. In Abb. 2 ist die Festigkeitsentwicklung über die Zunahme des Schwerweges schemenhaft dargestellt. Nach dem Erreichen des Peakwertes der Festigkeit erfolgt ein Abfall auf den Critical State und nach einer weiteren (orientierten) Bewegung und bei einem ausreichend großen Tonanteil der mögliche Abfall auf die sogenannte Restscherfestigkeit. Die Letztgenannte kann dabei auf Restwerte des Reibungswinkels von ca. $\phi^{\prime}=10^{\circ}$ abfallen [12].

Ein Beispiel für die "Streuung" der Scherparameter innerhalb einer vorab mittels Inklinometermessungen festgestellten Scherzone wurde in [16] ausgeführt. Für einen Rutschhang wurden die innerhalb einer Gleitzone ermittelten Scherparameter aus 3 Rahmenscherversuchen der rechnerischen Standsicherheitsuntersuchung des IstZustandes des Rutschhanges zugrunde gelegt. Dabei ergaben sich rechnerische Sicherheitszahlen von $\eta=0,78$ bis 1,18, was letztlich die gesamte Spannweite von einem "zufriedenstellenden Sicherheitszustand“ $(\eta=1,18)$ bis zu einem Zustand, bei dem das Versagen des Rutschkörpers schon hätte stattfinden müssen $(\eta=0,78)$, angibt. Daraus ist die Schwierigkeit einer Sicherheitsbeurteilung, alleine auf im Labor bestimmten Scherparameter basierend, erkennbar. Üblich ist es deshalb, dass für einen Rutschhang mit mehr oder weniger bekanntem Bewegungsverhalten durch den/die verantwortliche(n) Geotechniker/in eine Sicherheitszahl (zumeist auf Erfahrung beruhend) festgelegt wird, die geringfügig größer $\eta=1,0$ ist (i.A. zwischen ca. $\eta=1,0$ und 1,1$)$ und möglichst einer bekannten Bewe- gungsrate des Hanges zugeordnet werden kann. Davon ausgehend wird eine Rückrechnung der für diese abgeschätzte Sicherheitszahl zugeordneten Scherparameter ' 'und c' vorgenommen, wobei die Kohäsion c' zumeist aus Erfahrungswerten abgeschätzt wird. Basierend auf diesen rückgerechneten Parametern wird dann z.B. eine Beurteilung weiterer (baulicher) Maßnahmen zur Stabilisierung des Hanges hinsichtlich der Sicherheitszahl vorgenommen. Die im Weiteren ermittelten Sicherheitszahlen sind sodann weniger als Absolutwerte, sondern vielmehr als "Relativwerte", bezogen auf die ursprünglich vorgenommene Einschätzung der Sicherheitszahl für den Rutschhang in seinem natürlichen Zustand (Ist-Zustand), zu verstehen. Für große Massenbewegungen stellt dabei eine relative Erhöhung der Sicherheitszahl um einen Wert $\Delta \eta=0,03$ bis 0,06 (= 3 bis $6 \%$-Pkte) zumeist bereits eine ausreichende Sicherheitserhöhung dar, um einerseits eine signifikante Verringerung der mittleren jährlichen Bewegungsraten zu erreichen und andererseits die Wahrscheinlichkeit eines „plötzlichen Versagens“ mit ausreichender Sicherheit ausschließen zu können. Anzumerken ist, dass eine Sicherheitserhöhung im genannten Bereich bei "großen" Massenbewegungen (mit mehr als ca. 0,5 Mio $\mathrm{m}^{3}$ ) i.A. bereits mit enormen Kosten (z.B. > 1 Mio $€$ ) verbunden ist.

Eine andere (i.A. analytische) Herangehensweise zur Beurteilung des Zustandes von Rutschungen basiert auf der Annahme viskoser Stoffgesetze für die maßgebenden Bodenschichten. Beispielhaft seien hier jene von Lippomann 1988 [10] oder Puzrin \& Schmid 2012 [11] genannt. Diesen Ansätzen liegt die theoretische oder auf Ergebnissen von Laborversuchen beruhende Annahme zugrunde, dass die Schergeschwindigkeit von der aufgebrachten Scherspannung abhängt. In GI. 1 ist der Ansatz der geschwindigkeitsabhängigen Scherspannung nach Lippomann [10] dargestellt. In Abb. 4 sind die Modellansätze nach Lippomann und Puzrin \& Schmid dargestellt.

$$
\tau_{1}=\tau_{0} \cdot\left(1+I_{v \alpha} \cdot \ln \frac{v_{1}}{v_{0}}\right)
$$

Dabei stellt $I_{v a}$ den Viskositätsparameter, im gegenständlichen Ansatz den einzigen Bodenparameter, dar. Dieser Wert wurde von Lippomann [10] für feinkörnige Böden mit 3-6 \% angegeben. Für gemischtkörnige Böden, wie sie in Gleitzonen großer Massenbewegungen oftmals vorherrschen, hat sich, basierend auf Erfahrungswerten mit großen Rutschungen, ein Wert von ca. 0,75 bis $2 \%$ 
Abb. 4: Ansätze nach a) Häfeli [18], b) Lippomann [10], c) Puzrin \& Schmid [11]

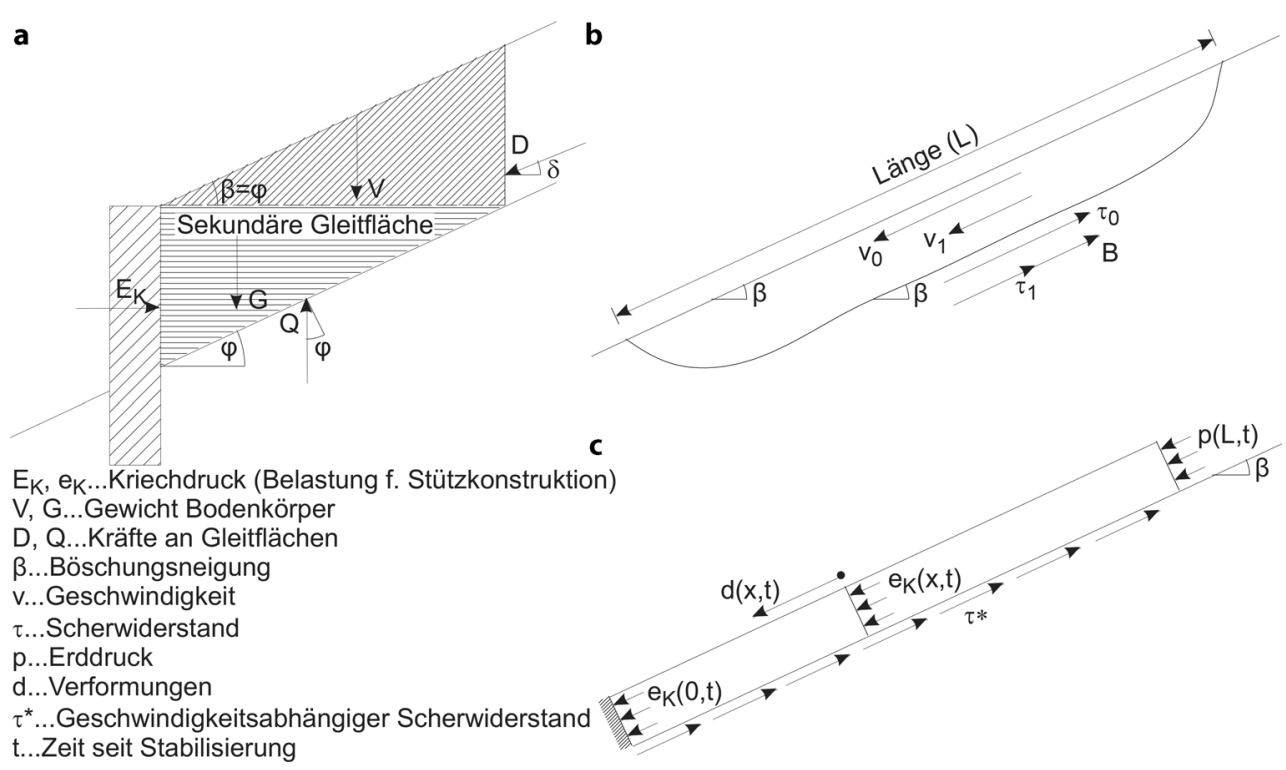

a

bewährt [17]. Da sich dieser Wert proportional auf die Bemessungskraft für allenfalls angedachte Stützmittel (durch die Reduktion der Scherspannung in der Scherfläche) auswirkt, ist eine möglichst genaue Bestimmung wichtig. Wie bereits weiter oben diskutiert, ist dies für große Rutschungen mit komplexen Untergrundverhältnissen mittels Laborversuchen kaum möglich. Zumindest in bestimmten Fällen kann er über Rückrechnungen ermittelt werden, wobei sich diese, im Vergleich zu oben angeführten Rückrechnungen zur Bestimmung der Sicherheitszahl $\eta$, i.A. deutlich schwieriger darstellen. In diesem Ansatz nach Lippomann kommt eine Sicherheitszahl nicht explizit zum Ausdruck. Der "Abstand“ zu einem möglichen kritischen Zustand ist über den Zusammenhang Schergeschwindigkeit zu Scherspannung, wie in Abb. 3 dargestellt, gegeben. Der in Abb. 3 dargestellte logarithmische Zusammenhang zeigt eine rasche Verlangsamung der Bewegungsraten durch einen geringen Stützmitteleinbau. Ein weiteres Abbremsen der Rutschung wird jedoch in überproportionaler Form aufwändiger. Die Bemessung von Stützmitteln zur Erhöhung der "Standsicherheit des Hanges" oder bei diesem Bemessungsansatz zutreffender ausgedrückt, zur Verringerung der Bewegungsraten der Rutschung, erfolgt derart, dass die zur Verringerung der Bewegungsrate des Hanges erforderliche Abnahme des Scherspannung über die Gleitfläche integriert, als Bemessungskraft für die Stützmittel herangezogen wird [10].

Eine weitere wichtige Größe und Grundlage bei der Auslegung und Bemessung von Stützmitteln (z.B. Pfahl- oder Brunnenwände, Ankerkonstruktionen) ist der Erddruck, welcher auf diese wirkt. Bei kleineren Rutschungen kann davon ausgegangen werden, dass durch die geplanten oder eingebauten Stützmittel ein vollkommener Stillstand der Rutschung erreicht werden kann. Der Erddruck, der als Bemessungsgröße auf die Stützkonstruktion anzusetzen ist, ermittelt sich dann in Bezug zur Gesamtmasse der Rutschung. Bei großen Rutschungen ist, wie oben bereits ausgeführt wurde, oftmals davon auszugehen, dass durch eingebaute Stützmittel eine Reduzierung der mittleren jähr- lichen Bewegungsraten, jedoch kein völliger Stillstand der Rutschmasse erreicht werden kann. In diesem Fall findet ein über die Zeit und mit Zunahme der Hangverschiebungen ansteigender Erddruck auf die Stützkonstruktion statt. Dieser Anstieg erfolgt so lange, bis entweder ein "Gleichgewichtszustand" ohne weitere Hangbewegungen erreicht wird, ein Versagen der Stützkonstruktion stattfindet (und es in Folge i.A. zu einer neuerlichen Zunahme der Bewegungsraten kommt) oder bis es zu einem „Überschieben" der Stützkonstruktion kommt. Der letzte Fall tritt ein, wenn der innere und äußere Widerstand der Stützkonstruktion selbst größer ist, als der Widerstand, der für das Überschieben der Konstruktion zu überwinden ist. Der im deutschsprachigen Raum bekannteste Ansatz für einen derartigen Grenzwert des Kriechdrucks stammt von Häfeli [18] und wurde in den 1980er-Jahren von Brandl/Dalmatiner [19] für praktische Anwendungen erweitert. In Abb. 4 sind die Ansätze nach Häfeli, Lippomann sowie Puzrin und Schmid in vereinfachter Weise dargestellt.

\section{Messtechnische Erkundungs- und Überwachungsmaßnahmen}

Klassische Erkundungsmaßnahmen wie Kernbohrungen, geophysikalische Untersuchungen etc. spielen bei der Untersuchung von Rutschungen, wie auch bei anderen Fragestellungen der Geotechnik, eine wesentliche Rolle. Im Gegensatz zu manch anderen Problemstellungen in der Geotechnik nehmen aber messtechnische „Erkundungs- bzw. Überwachungsmaßnahmen“ bereits in einem sehr frühen Stadium der Bearbeitung von Rutschungen eine besondere Rolle ein. Oftmals ist es erforderlich, bereits über Jahre vorauseilende messtechnische Überwachungen durchzuführen, bevor eine zutreffende Beurteilung der „Standsicherheit eines Rutschhanges " vorgenommen werden kann und/ oder mit einer zielgerichteten Planung von Sicherungsmaßnahmen begonnen werden kann. Dabei zielen diese vorlau- 

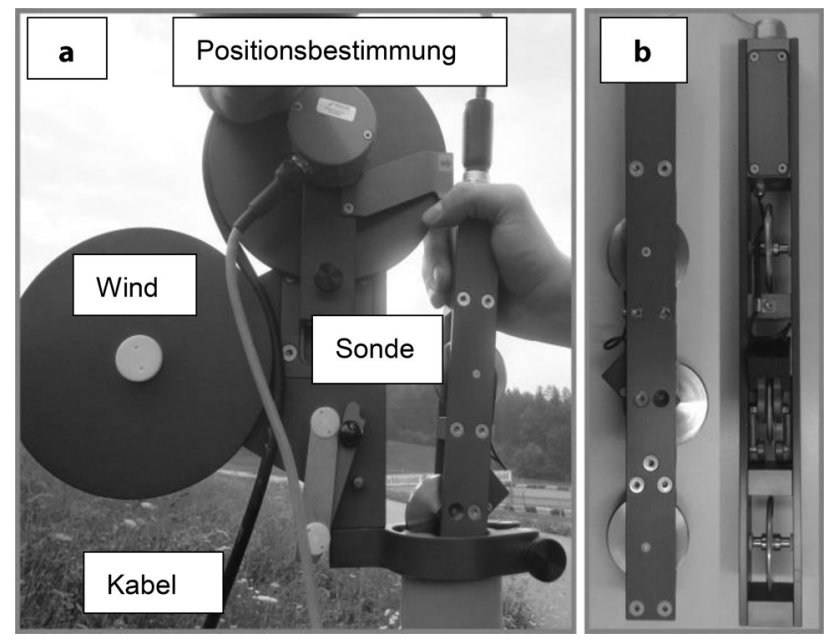

Abb. 5: Inclinodeformeter Messsonde

fenden Erkundungs- und Überwachungsmaßnahmen u.A. auf folgende Fragestellungen ab:

- Erfassung der Geometrie der Rutschmasse: Tiefe, Form und räumlicher Verlauf von Gleitflächen, Ausdehnung des Rutschungskörpers

- Bewegungsverhalten der Rutschmasse: Bewegungsraten über die Zeit sowie mögliche Korrelationen der Bewegungsraten mit äußeren Einflüssen („triggern“) wie z.B. Niederschlagsereignisse, Schneeschmelzphasen, Erschütterungen etc.

- Gezielte Untersuchung maßgebender Einflussgrößen: z.B. Daueraufzeichnung von Porenwasserdrücken in potentiellen Gleitflächen und Gegenüberstellung dieser Werte mit Bewegungsraten und/oder Niederschlagsereignissen.

Die Messmittel, die hierbei Anwendung finden, sind zahlreich und sprengen den Rahmen dieses Beitrags. Beispielhaft angeführt seien: Klassische geodätische Vermessungen, satellitenbasierte geodätische Vermessungen, Inklinometermessungen (klassisch oder Ketteninklinometer), Extensometermessungen, Porenwasserdruckmessungen, Wasserstandsmessungen oder Wassermengenmessungen (z.B. aus Drainagen oder Quellen), Aufzeichnungen von Wetterstationen über Niederschlag, Temperatur etc. Ein Überblick und eine Zusammenstellung derartiger Messverfahren findet sich z.B. in [20].

Den messtechnischen Überwachungsmaßnahmen kommt jedoch nicht nur in der Erkundungs- und Vorprojektierungsphase eine besondere Bedeutung zu, sondern bei großen Rutschungen insbesondere auch im Zuge sowie nach dem Einbau möglicher Sicherungsmaßnahmen. In vielen Fällen sind messtechnische Langzeit- und/oder Permanentüberwachungsmaßnahmen das am besten geeignete Mittel, um kritische Entwicklungen einer Rutschung erkennen und allenfalls erforderliche Maßnahmen (z.B. Evakuierung von Gefährdungszonen, Verstärkung von Stützmitteln etc.) einleiten zu können. Wie oben bereits ausgeführt, stellen sie oftmals die wesentliche Grundlage zur Sicherheits- bzw. Risikobeurteilung dar. Im Wesentlichen kommen dabei dieselben Messmittel, wie sie auch in der Vorerkundungsphase angesprochen wurden, zum Einsatz. Werden bei großen Rutschungen Stützmittel (wie z.B. vorgespannte Freispielanker in Kombination mit Betonstrukturen) zur Verlangsamung der mittleren Bewegungsraten eingebaut, so wird davon ausgegangen, dass weitere (planmäßig in Kauf genommene) Bewegungen des Hanges zu einer Zunahme der Beanspruchung dieser Stützmittel führen. Dies kann bis zum („planmäßigen“) Versagen solcher Stützmittel führen, sofern nicht vorher Verstärkungs- oder Entspannungsmaßnahmen für die Stützmittel vorgesehen werden [4]. In solchen Fällen spielen messtechnische Überwachungsmaßnahmen eine ganz besondere Rolle, da sie zur Beurteilung des Beanspruchungszustandes von Stützmitteln unbedingt erforderlich sind. Dabei kann die Beurteilung der Beanspruchung z.B. über Kraft- oder Spannungsmessungen (z.B. Kraftmessdosen von Ankern) oder über Verformungsmessungen (z.B. Inklinometermessungen) und darauf aufsetzende Rückrechnungen auf Spannungen oder allgemein Beanspruchungen in Tragelementen erfolgen [21]. Diese Vorgehensweise ist deshalb auch von besonderer Bedeutung, da die Prognostizierbarkeit der zeitlichen Entwicklung der Hangdeformationen, wie auch der daraus resultierenden Erddrücke auf Stützkonstruktionen bzw. allgemein der zeitlichen Entwicklung der Beanspruchung der Stützmittel i.A. schwierig und mit deutlichen Unschärfen behaftet ist. In Abb. 5 ist beispielhaft ein neuartiges Messinstrument - die sogenannte IDMSonde (Inclinodeformeter-Methode) dargestellt, mit Hilfe derer die Rohrdeformation (Ovalisierung des Rohrquerschnittes) eines klassischen Inklinometerrohres gemessen werden kann. Damit kann ein klassisches Inklinometerrohr nicht nur zur Verschiebungsmessung eines Rutschhanges herangezogen werden, sondern beispielsweise auch die Veränderung der Horizontalspannungen, die zu einer Querschnittsänderung des Inklinometerrohres führen, beurteilt werden [22].

Wird ein Inklinometerrohr beispielsweise bergseitig einer Stützkonstruktion eingebaut, kann aus der Querschnittsänderung des Rohres (welche mittels der IDM ermittelt werden kann) auf die Erddruckänderungen rückgerechnet werden. Daraus lässt sich die mögliche Erddruckzunahme auf die Stützkonstruktion über die Zeit, bis hin zur Feststellung eines sich abzeichnenden Versagens der Stützkonstruktion (wenn keine weitere oder gegenläufige Ovalisierung des Rohrquerschnittes gemessen wird) feststellen. Diese neuartige Messmethode konnte schon bei verschiedenen Fallbeispielen von Baugruben wie auch Rutschungen erfolgreich angewendet werden [23, 24].

\section{Kurze Anmerkung zu Sicherungs- und Sanierungsmaßnahmen für Rutschungen}

Hinsichtlich der Vielzahl an möglichen Sicherungsansätzen für Rutschungen wird auf [12] verwiesen. Zum Abschluss des gegenständlichen Beitrages wird noch auf einige Gesichtspunkte hingewiesen, die bei der Wahl von Sicherungsmaßnahmen i.A. zu berücksichtigen sind. 
Grundsätzlich ist das Ziel einer Sicherung vorab zu definieren. Dies beinhaltet einerseits die Festlegung der (hauptsächlich) zu schützenden Objekte wie z.B. spezielle Bauwerke auf dem Rutschungskörper oder unterhalb von diesem. Die Hauptausrichtung der Sicherungsmaßnahme(n) bestimmt i.A. die Art der Sicherungs- bzw. Stützmittel, aber zumeist auch die Lokalität, an der diese angebracht werden. Im Fußbereich einer (großen) Rutschung eingebaute Stützmittel haben zwar für den unteren Rutschungsbereich eine "rasche" positive Auswirkung, für den oberen Rutschungsbereich kann es jedoch "lange“ dauern, bis sich eine deutliche Verzögerung der Bewegungsrate der Rutschung in diesem Bereich einstellt. Dieser "Verzögerungseffekt " kommt beispielsweise in dem oben bereits vorgestellten Modellansatz von Puzrin [11] zum Ausdruck. Befinden sich die hauptsächlich zu schützenden Objekte somit im oberen Rutschungsbereich, ist i.A. zumindest ein Teil der Sicherungsmittel in diesem Bereich des Rutschungskörpers einzubauen.

Hinsichtlich der Art der Stütz- bzw. Sicherungsmittel kann einerseits in konstruktive Maßnahmen zur Erhöhung der stützenden Kräfte und andererseits in Maßnahmen, die in erster Linie auf die Verringerung der "treibenden" Kräfte (wie z.B. Entwässerungsmaßnahmen oder Entspannungsbohrungen zur Reduzierung von Porenwasserdrücken) hinwirken, unterschieden werden. Bei den konstruktiven Maßnahmen zur Erhöhung der stützenden Kräfte ist die Unterscheidung von weitestgehend "steifen" (im Extremfall starren und damit in Zusammenhang stehend zumeist eher "spröden“) Elementen und "flexiblen“ bzw. "duktilen" Systemen vorzunehmen. Für "kleinere bis mittelgroße Rutschungskörper", die durch die konstruktiven Stützmittel zum Stillstand gebracht werden können (und zumeist auch sollen), weisen eher steife Stützmittel wie z.B. (verankerte) Pfahlwände oder Stützscheiben den Vorteil auf, dass sie praktisch "sofort" bzw. innerhalb sehr kurzer Zeit wirken und somit zu einem raschen Stillstand der Rutschung führen. Für (sehr) große Rutschungskörper, deren Bewegungen aus wirtschaftlichen und oftmals auch technischen Gründen per (Ziel-)Definition nicht vollständig gestoppt werden (können), weisen „nachgiebige bzw. flexible“ Konstruktionen die folgenden Vorteile auf. Einerseits kann die Bemessungskraft (aus dem wirkenden Erddruck) geringer als für "starre Konstruktionen“ gewählt werden. Auf diesen Umstand verweisen z.B. Brandl \& Dalmatiner in [19]. Des Weiteren geht dies auch aus Bemessungsansätzen mit viskosen Stoffmodellen, wie sie oben diskutiert wurden [10] und [11] hervor, da davon ausgegangen wird, dass die Rutschung auch nach der Sanierung noch eine gewisse Bewegungsrate aufweist und somit höhere Scherwiderstände in der Gleitfläche wirken, als dies bei einer zum Stillstand gebrachten Rutschung der Fall ist. Bei zu steifen (starren) Stützkonstruktionen kommt es somit zu einem rascheren und größeren Anstieg des Erddruckes, der besonders bei sehr großen Rutschungen und sich eher spröd verhaltenden Bauwerken zu einem frühzeitigen Versagen dieser Konstruktion führen kann. Eine sehr flexible und in verschiedener Hinsicht sehr anpassungsfähige Stützkonstruktion zur Sicherung von Lockergesteinsrutschungen sind Betonelemente, die mit vorgespannten Freispielan- kern in den stabilen Untergrund verankert werden. Diese weisen bei richtiger Auslegung die geforderte hohe Nachgiebigkeit, einen mit zunehmenden Hangbewegungen zunehmenden Widerstand und eine entsprechende Adaptionsmöglichkeit beim Erreichen des Grenzwertes der Tragfähigkeit der Anker auf. Einerseits können die Anker durch bestimmte, bereits bei der Herstellung zu berücksichtigende Maßnahmen, entspannt werden, andererseits kann eine derartige Konstruktion bei Erfordernis sehr gut erweitert werden (z.B. durch die Anbringung zusätzlicher verankerter Betonelemente). Ein derartiges Fallbeispiel wurde z.B. in [4] präsentiert.

Bei technischen Maßnahmen, die auf eine Verringerung der treibenden Kräfte abzielen, sind als häufigste Form Entwässerungsmaßnahmen oder Entspannungsmaßnahmen für die Reduzierung von Porenwasserdrücken in den Gleitschichten zu nennen. In manchen Fällen reichen derartige Maßnahmen alleine zur Sicherung bzw. Sanierung einer Rutschung aus. Doch obwohl es oftmals möglich ist, die Wasserverhältnisse im Untergrund als den wesentlichen Auslöser für eine Rutschung auszumachen, ist es dennoch nicht immer möglich, mit einfachen technischen Mitteln (z.B. Entwässerungsbohrungen) eine ausreichende Entwässerung oder Porenwasserdruck-Entspannung sicherzustellen. Es ist auch darauf zu achten, dass Entwässerungsmaßnahmen (z.B. eingebaute Drainagerohre) bei weiter stattfindenden Hangbewegungen abreißen können und es in Folge zu einer Bewässerung der Gleitfläche (durch bergseitig von dieser gefassten Wässer) kommen kann.

Open access funding provided by Graz University of Technology.

Open Access Dieser Artikel wird unter der Creative Commons Namensnennung 4.0 International Lizenz (http://creativecommons.org/licenses/ by/4.0/deed.de) veröffentlicht, welche die Nutzung, Vervielfältigung, Bearbeitung, Verbreitung und Wiedergabe in jeglichem Medium und Format erlaubt, sofern Sie den/die ursprünglichen Autor(en) und die Quelle ordnungsgemäß nennen, einen Linkzur Creative Commons Lizenz beifügen und angeben, ob Änderungen vorgenommen wurden.

\section{Literatur}

1. Rutschungstypen (Multilingual Landslide Glossary): Ernst \& Sohn: Grundbautaschenbuch, 6. Auflage 6,(1993), Teil 1: Geotechnische Grundlagen, Kapitel 1.13, Seite 636, Bild 2

2. Latif, M.: Klimawandel und Klimadynamik; Stuttgart: Ulmer, 2009 , S. $180 \mathrm{f}$

3. Hungr, O.: A review of landslide hazard and risk assessment methodology; Landslide and Engineered Slopes - Experience, Theory and Practice - Aversa et al (Editors), Proceedings of the 12th International Symposium on Landslides, Napoli, Italy, June 2016

4. Marte, R.: Gutsche, R.: Rutschungssanierung B90 - Doppelkehre Nassfeld; Beiträge zu den Niederösterreichischen Geotagen, 2007

5. Marte, R.; Garber, E.; Edlmair, G.: Errichtung eines Autobahntunnels in offener Bauweise am Fußbereich eines instabilen Hanges. 20. Christian Veder Kolloquium, Graz, 2005

6. Forstinger+Stadlmann ZT OEG: Geotechnisches Beobachtungsprogramm ("Monitoring“) Graukogel Nordwesthang Bad Gastein. Baugeologisch-geotechnische Bearbeitung; Unveröffentlichtes Gutachten, 2005

7. Ebner, M.: Geotechnisch-geologische Untersuchung einer großen, kriechenden Massenbewegung am Beispiel Graukogel in Bad Gastein; Masterarbeit, Technische Universität Graz, 2013 
8. Lotter, M.; Moser, M.: Die Massenbewegungen der Naßfeldregion; Abhandlungen der Geologischen Bundesanstalt, Band 61 (2007), S 159-173; Wien

9. Marte, R.; Moyschewitz, G.; Harml, J.: Construction of a snow retention basin in an Alpine sagging mass; Geomechanics and Tunnelling, 7 (2014), Nr. 4, S $328-338$

10. Lippomann, R.: Ingenieurgeologische Kriechhangsicherung durch Dübel. Dissertation, Universität Karlsruhe, 1988

11. Puzrin, A.M.; Schmid, A.: Evolution of stabilized creeping landslide, Géotechnique 62 (2012), No. 6, pp. 491-501. ICE Publishing: London, 2012

12. Brandl, H.: Grundbau-Taschenbuch, 7. Auflage, (2009), Teil 3: Gründungen und geotechnische Bauwerke; Kap. 3.9 Stützbauwerke und konstruktive Hangsicherungen

13. Atkinson, J.: Shear strength. GeotechniCAL. http://environment. uwe.ac.uk/geocal/SoilMech/shear/shear.htm. (Accessed 08 October 2013)

14. Skempton, A.W.: Residual strength of clays in landslides, folded strata and the laboratory, Géotechnique 35 (1985), No. 1, pp. 3-18. ICE Publishing: London, 1985

15. Wood, D.M.; Soil behaviour and critical state soil mechanics; Cambridge University Press, 1990

16. Marte, R.; Ausweger, G.: Safety Definition and Estimation for Slopes with Creep Behaviour - A Geotechnical Challenge; Vortrag beim 62. Geomechanik Kolloquium, Salzburg, 09.10.2013
17. Marte, R.; Ausweger, G.: Modellierung des Verhaltens von Kriechhängen; Der Bauingenieur 89 (2014), Februar, S. 79 - 88

18. Haefeli, R.: Zur Erd- und Kriechdrucktheorie. Mit einer Anwendung auf den Castieler Viadukt der Linie Chur-Arosa der Rhätischen Bahn. Mitteilungen aus der Versuchsanstalt für Wasserbau an der Eidgen. Techn. Hochschule, 1945

19. Brandl, H.; Dalmatiner, J.: Brunnenfundierungen von Bauwerken in Hängen (insbesondere Brücken), Straßenforschung, Heft 352 (1988)

20. Boley, C. (Hrsg.): Handbuch der Geotechnik, Grundlagen - Anwendungen - Praxiserfahrungen; Springer Vieweg, 2012

21. Marte, R.: Rückrechnung der Schnittgrößen und Belastung von horizontal beanspruchten Stahlbetonpfählen zur Sicherung von Hangrutschungen aus Verformungsmessungen (Dissertation), Gruppe Geotechnik Graz, Heft 2 (1998), Fakultät für Bauingenieurwesen, Technische Universität Graz

22. Schwager, M. : Development, analysis and applications of an 'inclinodeformeter' device for earth pressure measurements; Dissertation; ETH Zürich, 2013

23. Ausweger, G.: Earth pressure on retaining walls in slopes; Proceedings of the $23^{\text {rd }}$ European Young Geotechnical Engineers Conference, Barcelona, 2014

24. Schwager, M.V.; Puzrin, A.M.: Inclinodeformeter pressure measurements in creeping landslides: analytical solutions and field applications, Géotechnique 64 (2014), No. 6, S. 447-462 